\title{
CONOCIMIENTO, USO Y VALORACIÓN DE LA FLORA VASCULAR DE TIERRA DEL FUEGO: EL EJEMPLO DE KARUKINKA
}

\author{
KNOWLEDGE, USE AND VALUATION OF VASCULAR PLANTS FROM TIERRA DEL FUEGO: \\ THE EXAMPLE OF KARUKINKA
}

Cora Menegoz ${ }^{1,2,3}$, Claudia Cerda ${ }^{4} \&$ Bárbara Saavedra $^{3}$

\begin{abstract}
Biodiversity conservation requires knowledge and valuation of the preserved entity. Wildlife Conservation Society (WCS) is leading a conservation program in southern Tierra del Fuego, in the private park Karukinka that expects to preserve globally important ecosystems for the benefit of the present and future, local and global community. The overall objective of this research is to know the current and potential use of plants contained in Karukinka, to determine the degree of knowledge among local people on this flora and their uses, as well as the level of valuation of the latter. Therefore, we expect to contribute to the valuation of the biota of the area, making suggestions for future environmental education programs, possible to be implemented by WCS. This work has evaluated the potential use for vascular plants of Karukinka through an exhaustive checking of botanical, ethnobotanical and others kinds of literature. Current use was estimated using specific surveys on the population of the area of Porvenir and Punta Arenas. The polls were conducted in two strata of the Magallanes region population: schoolchildren and professionals. These surveys dealt with the knowledge about vascular plants of Tierra del Fuego, its uses, and their valuation. They were analyzed combining qualitative and quantitative methods with general descriptive statistics. The survey results confirm the initial hypothesis of the existence of a large gap of knowledge about flora of Tierra del Fuego, especially alarming in the case of schoolchildren and adults of Porvenir.
\end{abstract}

Key words: Ethnobotanics, Conservation, Environmental education, Patagonia, Plants.

\footnotetext{
${ }^{1}$ Facultad de Agronomía e Ingeniería Forestal, Pontificia Universidad Católica de Chile. ko.menegoz@gmail.com

${ }^{2}$ Ecole d'ingenieurs en agronomie de Purpan, Toulouse, Francia.

${ }^{3}$ Wildlife Conservation Society (WCS), Santiago, Chile.

${ }^{4}$ Facultad de Ciencias Forestales y Conservación de la Naturaleza, Universidad de Chile.
} 


\section{RESUMEN}

La preservación de la biodiversidad requiere del conocimiento y valoración del objeto de conservación. Wildlife Conservation Society (WCS) se encuentra liderando un programa de conservación en el parque privado Karukinka, al sur de Tierra del Fuego, donde se espera preservar ecosistemas vegetales de importancia mundial, en beneficio de la comunidad local y global, presente y futura. El objetivo general de esta investigación es conocer el uso actual y potencial de la flora vascular contenida en Karukinka, determinar el grado de conocimiento de la población local, así como los usos que se le reconocen. Asimismo, se espera conocer el nivel de valoración de la flora vascular de Tierra del Fuego. Se espera con esto contribuir a la conservación de la biota presente en la zona, a través de la definición de propuestas específicas para futuros programas de educación ambiental, posibles de ser implementados por WCS. Este trabajo evaluó el uso potencial de la flora vascular de Karukinka a través de una revisión exhaustiva de literatura botánica, etnobotánica y otras. El uso actual se estimó aplicando encuestas específicas en la población de la zona de Porvenir y Punta Arenas. Las encuestas fueron realizadas en dos estratos de la población de la Región de Magallanes: escolares y profesionales, con las cuales se evaluó el grado de conocimiento de las plantas vasculares de Tierra del Fuego, sus usos conocidos, y su valorización. Las encuestas fueron analizadas cualitativa y cuantitativamente utilizando estadística descriptiva general. El resultado de las encuestas confirma la hipótesis inicial de la existencia de un vacío importante de conocimiento respecto de la vegetación de Tierra del Fuego. Esta brecha resulta especialmente alarmante en el caso de los escolares de ambas localidades y de los adultos de Porvenir.

Palabras clave: Etnobotánica, Conservación, Educación ambiental, Patagonia, Vegetación.

\section{INTRODUCCIÓN}

Como la mayoría de los países latinoamericanos, Chile es un país que se desarrolló rápidamente gracias a la explotación de sus recursos naturales, siendo este desarrollo poco controlado y en general negativo para los ecosistemas nativos (De Gregorio, 2009; Otero, 2006). En los últimos 20 años sin embargo, desde la promulgación de la Ley № 19.300 sobre Bases Generales del Medio Ambiente en 1994, que fue modificada en 2010 por la Ley № 20.417, la gestión ambiental de Chile ha demostrado avances con distintas formas e iniciativas de conservación (Ministerio de Agricultura, 2008; Lara, 2009). En este contexto, el parque privado Karukinka, proyecto recientemente emprendido por la Wildlife Conservation Society (WCS), se esfuerza por conservar y promover el uso sostenible de la biodiversidad en el sur de Tierra del Fuego. Este lugar presenta un alto valor cultural, por haber sido el hogar de los indígenas onas, una etnia hoy día desaparecida, y un alto valor ecológico, por la gran diversidad de ecosistemas que contiene (WCS Chile, 2009).
La etnobotánica es una disciplina científica relativamente nueva, que según Ocampo (1994) se define como el estudio de las relaciones recíprocas entre los grupos humanos y la vegetación que los rodea. Numerosas publicaciones han demostrado la importancia de los estudios etnobotánicos en la conservación biológica y el manejo de los recursos vegetales (Castetter, 1944; Fischer \& Van der Wal, 2007; Fischer \& Young, 2007; Albuquerque et al., 2009). El vínculo entre el conocimiento tradicional de las plantas, la percepción que tiene la comunidad sobre estas y la conservación de la biodiversidad, ha recibido cada vez más atención de la comunidad científica durante las últimas décadas debido a la creciente pérdida del conocimiento tradicional y la degradación de hábitats naturales (Bermúdez et al., 2005). Los estudios etnobotánicos presentan un papel importante en la conservación proponiendo modelos funcionales para el uso y manejo sustentable de los recursos naturales, aplicables a las decisiones de protección y desarrollo (Albuquerque et al., 2009).

Uno de los aspectos que se espera desarrollar en Karukinka se refiere al análisis del conocimiento, uso y valoración de su vegetación, nociones relacionadas 
con la disciplina de la etnobotánica (Ladio, 2007). Esto es especialmente relevante, considerando la importante presencia humana que tuvo Tierra del Fuego en el pasado (Chapman, 1986), el atractivo mundial que hoy día esta zona concita (Sernatur, 2011), y la necesidad de desarrollar instrumentos de manejo que faciliten la conservación de las especies vegetales en el futuro (Secretaría del Convenio sobre la Diversidad Biológica, 2009).

El objetivo general de esta investigación es conocer el uso actual y potencial de la flora contenida en Karukinka, determinar su grado de conocimiento entre la población local, así como los usos que se le reconocen. Asimismo, se espera conocer el nivel de valoración de la flora de Tierra del Fuego. El discernimiento de la brecha de conocimiento local sobre la flora contenida en Karukinka podría ser una herramienta clave para guiar el diseño futuro de proyectos de educación para la conservación en la zona.

La hipótesis inicial considera que el nivel de conocimiento y valoración de la flora vascular en las poblaciones locales de Tierra del Fuego es pobre, reflejo del bajo interés del país y de su población en asuntos medio ambientales (Universidad de Chile, 2000), así como al escaso acceso a la información, ya sea a través de medios de enseñanza formal $e$ informal. En términos comparativos, se espera que la población presente en Tierra del Fuego, más directamente relacionada con Karukinka, y con un menor grado de urbanización, pueda presentar un conocimiento y valoración mayor de la vegetación de la Isla, en comparación a la población de Punta Arenas, la urbe más importante de la Región.

\section{MATERIALES Y MÉTODOS}

Sitio de estudio

El estudio se realizó en las dos ciudades más pobladas próximas al parque Karukinka: Porvenir y Punta Arenas.

Punta Arenas es la capital de la Región de Magallanes y Antártica chilena. Esta ciudad cuenta con 130.704 habitantes, lo que corresponde a 82\% de la población regional (INE, 2012). Del total de la población, 97\% es urbana. Al estar ubicada en el Estrecho de Magallanes, Punta Arenas logró su desarrollo siendo el principal puerto de navegación entre los océanos Pacífico y Atlántico antes de la apertura del Canal de Panamá en 1920 (SUBDERE, 2009a).

Por otra parte, la comuna de Porvenir se encuentra al este del Estrecho de Magallanes, en la Isla Grande de Tierra del Fuego. Pertenece, junto a las comunas de Primavera y Timaukel, a la Provincia de Tierra del Fuego, Región de Magallanes y Antártica chilena. Porvenir es, a su vez, la Capital provincial (SUBDERE, 2009b). Cuenta con 5.907 habitantes, lo que representa el $84 \%$ de la población total de la Provincia de Tierra del Fuego y 3,7\% de la población regional. De los 5.907 habitantes, 5.390 viven en la zona urbana, lo que corresponde a $91,2 \%$ de la población (INE, 2012).

\section{Revisión bibliográfica}

Durante los dos primeros meses del estudio, se realizó una amplia investigación bibliográfica sobre los 424 taxones de flora vascular presentes en Karukinka, buscando los distintos tipos de uso y nombres que el ser humano haya aplicado en el pasado o en la época actual, para cada planta. La mayoría de las fuentes de información utilizadas fueron primarias, correspondiendo a libros, artículos de revistas científicas provenientes de bases de datos especializadas (JSTOR, Web of Science e ISI Web of Knowledge), y sitios web relacionados con el uso de las plantas.

Además de representar el gran valor de la flora vascular de Karukinka, esta información constituye una referencia para este trabajo, una base para poder analizar las encuestas realizadas en las comunidades locales y apreciar si existe o no una brecha de conocimiento.

Concepción de la herramienta de observación

Para este trabajo se optó por utilizar cuestionarios, a fin de analizar cuantitativamente las tendencias poblacionales. Con este método se puede lograr un $\mathrm{N}$ muestral relativamente amplio en un tiempo limitado (Quivy \& Van Campenhoudt, 2006).

Se realizaron dos tipos de cuestionarios: a) Destinado a escolares entre 13 y 18 años y b) Destinado a funcionarios públicos y fiscales.

Las encuestas estuvieron constituidas por 
cuatro dimensiones: a) Conocimiento general sobre las plantas vasculares de Tierra del Fuego; b) Conocimiento sobre el uso de las plantas y el efecto de este uso sobre la naturaleza; c) Valoración de los distintos tipos de plantas (e.g. nativas, exóticas, en peligro de extinción, utilizadas o no); d) Datos generales sobre la persona (e.g. edad, sexo, desde cuanto tiempo vive en la región).

La primera de estas dimensiones estuvo orientada a indagar en el conocimiento de las plantas vasculares, donde se introdujo el cuestionario con una presentación de las plantas vasculares. El encuestado tuvo que indicar nombres de plantas (árboles, arbustos, hierbas y helechos), qué sabía de ellas, cómo las había conocido, y en qué lugar pensaba poder encontrarlas. Un ejercicio de reconocimiento de plantas a partir de fotos: una planta muy común, el calafate (Berberis microphylla G. Forst.), y otra planta menos conocida, también fue incorporado.

La segunda parte del cuestionario estuvo orientado a evaluar la dimensión sobre el uso de las plantas. El encuestado debió clasificar distintos tipos de usos según su nivel de importancia, dar tres ejemplos de plantas utilizadas por él o por personas de su entorno, un ejemplo de planta que haya sido utilizada antiguamente por los indígenas onas (Selk'nam) o yaganes (Yámana), y su opinión sobre el impacto del uso de las plantas sobre la naturaleza.

Por último, se indagó en la dimensión relativa a la valorización de las plantas. Las primeras preguntas de esta sección se enfocaron en la afición de los participantes por el contacto con la naturaleza. Luego se presentaron tres plantas - una vulnerable, una endémica y una exótica-y se preguntó si era importante o no conservar estas plantas en Tierra del Fuego. Las dos últimas preguntas ponían el encuestado en situaciones de decisión entre conservar una planta exótica o una nativa; y entre conservar Drimys winteri J.R. Forst. \& G. Forst., una planta muy utilizada en Chile y que ha sido utilizada durante varios siglos por los indígenas, o Crassula moschata G. Forst., una planta muy poco conocida y poco utilizada.

Además, cada dimensión contenía escalas de Likert con enunciados o afirmaciones (ítems), sobre los que manifestaba el individuo respecto a su valoración, escogiendo un nivel de acuerdo desde lo más favorable (totalmente de acuerdo) a lo más desfavorable (totalmente en desacuerdo).
El nivel de conocimiento de la vegetación de Tierra del Fuego se analizó entregando cuestionarios a escolares y a funcionarios públicos y fiscales que trabajan en áreas directa o indirectamente relacionadas con el ámbito de la conservación en esta zona. Estos dos estratos poblacionales son importantes para la conservación, ya que los niños representan hoy día a las futuras generaciones, de las cuales depende la sustentabilidad de los proyectos de conservación, mientras que los funcionarios son agentes de comunicación, financiamiento, tomadores de decisión, etc., para la conservación hoy en día.

\section{Diseño muestral}

Los cuestionarios combinaban distintos tipos de preguntas, en un formato claro, preciso y de fácil comprensión. Las preguntas abiertas, especialmente enfocadas al nivel del conocimiento, permitieron indagar nombres y usos de plantas, $y$ dejar a las personas expresarse libremente sobre un tema. Este tipo de pregunta conduce a un análisis más bien cualitativo (Quivy \& Van Campenhoudt, 2006). Por otra parte, se formularon preguntas cerradas, escalas nominales, ordinales, y escalas de actitud tipo Likert, las cuales son utilizadas en la medición de actitudes (Elejabarrieta \& Iñiguez, 1984). Estos tipos de preguntas limitan la expresión de las personas interrogadas, ya que el encuestado debe escoger entre respuestas preestablecidas, permitiendo realizar análisis cuantitativos (Seltiz et al., 1965).

Una validación de los cuestionarios se realizó en la ciudad de Santiago, en un grupo independiente de escolares y de adultos.

Las encuestas fueron diseñadas y dirigidas hacia cuatro grupos: escolares (30 personas por localidad) y funcionarios públicos (14 personas por localidad).

\section{Recolección de información en terreno}

El trabajo en terreno se efectuó durante las dos primeras semanas de noviembre del año 2009 en las ciudades de Punta Arenas y Porvenir. Los colegios donde se entrevistaron estudiantes son: Liceo Experimental UMAG en Punta Arenas y Liceo Hernando de Magallanes en Porvenir; y las instituciones públicas que estuvieron de acuerdo anteriormente con participar en el estudio. El 
cuestionario fue enviado por correo electrónico a la mayoría de los funcionarios públicos para que lo pudieran contestar con antelación. Los escolares contestaron el cuestionario en clase, supervisados por dos adultos.

\section{Análisis estadístico}

Los análisis cuantitativos se efectuaron en base a herramientas de estadística descriptiva simple. Se realizó un análisis de frecuencias destinados a sintetizar la información recopilada, especialmente de aquellas variables nominales y ordinales (Azócar de la Cruz, 2009). Los resultados de las escalas ordinales, nominales y de las preguntas abiertas fueron procesados y agrupados de manera de simplificarlos para poder realizar una interpretación cuantitativa. Los nombres de plantas citadas quedaron como información cualitativa, seleccionando solamente las plantas mayormente citadas. Se utilizaron frecuencias relativas para comparar dos o más distribuciones de frecuencias de muestras de tamaños diferentes, ordenando los resultados en tablas de distribución de frecuencias. El $\mathrm{N}$ de cada muestra varió según si se consideraba el número de personas o el número de citas de plantas hechas por ellas.

\section{RESULTADOS}

Revisión bibliográfica

En Karukinka se han registrado un total de
59 plantas vasculares que presentan uso múltiple (i.e. 5 ó más tipos de uso), es decir 14\% del total de la flora. 35\% de estas plantas son introducidas. Los usos mayormente representados en la flora vascular de Karukinka, considerando los usos pasados, presentes y potenciales (cuando la planta ha sido utilizada en otra parte del mundo), incluyen: forrajero, medicinal y químico, ornamental y alimenticio. Las familias mayormente usadas son Apiaceae, Asteraceae, Berberidaceae, Caryophyllaceae, Chenopodiaceae, Fabaceae, Fagaceae, Geraniaceae, Labiatae, Polygonaceae, Ranonculaceae, Rosaceae, Urticaceae y Poaceae. De las especies consideradas, 3\% están clasificadas en categorías de conservación.

Características de los grupos encuestados

Se encuestó un total de 88 personas entre escolares $(n=60)$ y adultos $(n=28)$ en las localidades de Punta Arenas y Porvenir. La edad media de los escolares de Punta Arenas es de 15 años, y la de los de Porvenir de 15,5 años. En el caso de los adultos, la edad media es de 37 años en Punta Arenas y de 40,5 años en Porvenir (Tabla 1).

La permanencia en la Región, el origen y el nivel de educación de las personas, muestra que el grupo de adultos de Punta Arenas es distinto a su homólogo de Porvenir, presentando menor tiempo en la región (promedio de 12,9 años en Punta Arenas v/s 23,4 años en Porvenir), menor porcentaje de personas originarias y mayor nivel de estudios (100\% universitario en Punta Arenas v/s 28,6\% en Porvenir) (Tabla 1).

TABLA 1: Descripción general de los grupos encuestados en Punta Arenas y Porvenir, Región de Magallanes, Chile.

\begin{tabular}{|c|c|c|c|c|c|}
\hline & \multicolumn{2}{|c|}{ Escolares } & \multicolumn{2}{|c|}{ Adultos } \\
\hline & & $\begin{array}{l}\text { Punta Arenas } \\
(\mathrm{N}=30)\end{array}$ & $\begin{array}{c}\text { Porvenir } \\
(\mathrm{N}=30)\end{array}$ & $\begin{array}{l}\text { Punta Arenas } \\
\quad(\mathrm{N}=14)\end{array}$ & $\begin{array}{l}\text { Porvenir } \\
(\mathrm{N}=14)\end{array}$ \\
\hline \multirow{2}{*}{ Sexo (\%) } & $\mathrm{F}$ & 56,7 & 73,3 & 42,9 & 50 \\
\hline & M & 43,3 & 26,7 & 57,1 & 50 \\
\hline \multicolumn{2}{|c|}{ Edad [Media (Rango)] } & $15(13-18)$ & $15,5(13-18)$ & 37 (20-60 y más) & 40,5 (20-60 y más) \\
\hline \multicolumn{2}{|c|}{ Domicilio en zona urbana (\%) } & 100 & 100 & 85.7 & 100 \\
\hline \multicolumn{2}{|c|}{$\begin{array}{l}\mathrm{N}^{\circ} \text { años en la Región } \\
\text { [Media (Rango)] }\end{array}$} & $10(1-18)$ & $11,8(1-17)$ & $12,9(1-39)$ & $23,4(3-51)$ \\
\hline \multicolumn{2}{|c|}{ Originarios de Magallanes (\%) } & 66,7 & 66,7 & 28,6 & 57,1 \\
\hline
\end{tabular}


Conocimiento de la vegetación de Tierra del Fuego

En cuanto al conocimiento de la vegetación de Tierra del Fuego, se observa una gran diferencia entre ambas localidades a nivel de adultos. Aquellos de Punta Arenas se muestran más de acuerdo en cuanto al conocimiento y aprendizaje sobre plantas (Fig. 1), específicamente 64,3\% de ellos se muestra de acuerdo con la frase "En mi vida cotidiana aprendo bastante sobre naturaleza y plantas" y $42,9 \%$ se muestra de acuerdo con la frase "Yo sé mucho de plantas vasculares de mi región porque tengo contacto con ellas muy a menudo". No así los adultos de Porvenir que se muestran de acuerdo sólo en un $14,3 \%$ y $7,1 \%$ respectivamente. En cuanto a los escolares, en ambas localidades entre un 50 y 60\% de ellos se muestra en desacuerdo (Fig. 1).

El conocimiento sobre hierbas en la población es escaso, observándose un elevado porcentaje de respuestas nulas respecto a citas de hierbas. Más de $80 \%$ de los escolares no citan ninguna hierba. Errores comunes entre los entrevistados incluyen la cita de árboles o arbustos en vez de hierbas, incluyendo especies como matico (Buddleja globosa Hope), calafate (Berberis microphylla G. Forst.), quintral (Tristerix tetrandus (R. et P) Martius), boldo (Peumus boldus Molina), rosa mosqueta (Rosa eglanteria L.), helechos, zarzaparrilla (Ribes magellanicum Poir.) y chaura (Gaultheria spp.) como parte del estrato herbáceo.

Tal como en el caso de las hierbas, se constata un escaso conocimiento de especies de helechos en

\section{En mi vida cotidiana/colegio aprendo bastante sobre naturaleza y plantas}

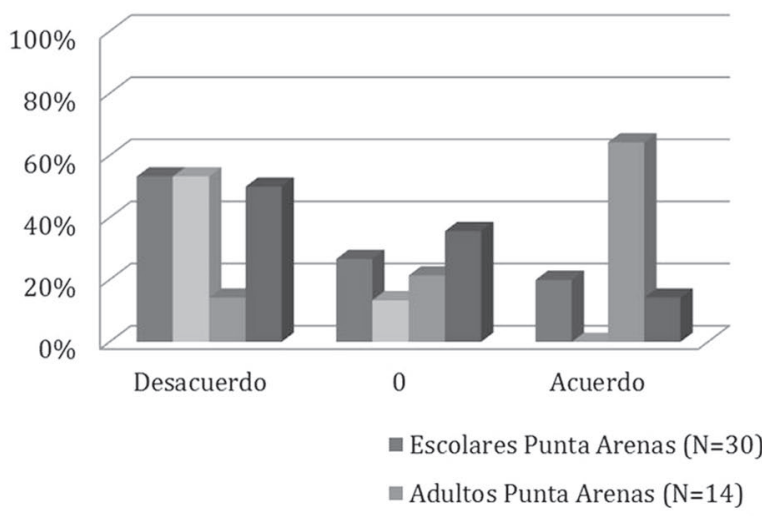

los grupos encuestados. Ningún escolar es capaz de nombrar una sola especie de helecho y sólo unos pocos adultos de Porvenir ( $\mathrm{N}=1)$ y Punta Arenas $(\mathrm{N}=2)$, nombran especies de helechos correctamente. Las especies más citadas son las del género Blechnum.

El conocimiento sobre arbustos entre la población encuestada es igualmente escaso. Una proporción importante de individuos no cita ningún arbusto, especialmente escolares en comparación con los adultos (70\% v/s 21,4\%). Los arbustos citados 2 ó más veces por escolares son calafate (Berberis microphylla G. Forst., nativo) y frambuesa (Rubus idaeus L., introducida), mientras que los adultos citan especialmente especies nativas incluyendo calafate y michay (Berberis ilicifolia L.f.), mata negra (Chiliotrichum diffusum (G. Forst.) Kuntze o Mulguraea tridens (Lag.) N. O'Leary \& P. Peralta), murtilla (Empetrum rubrum Vahl ex Willd.), chaura (Gaultheria mucronata (L. f.) Hook. et Arn.), romerillo (Baccharis patagonica Hook. \& Arn. o Chiliotrichum diffusum (G.Forst.) Kuntze) y zarzaparrilla (Ribes magellanicum Poir.).

El patrón de conocimiento observado para árboles es similar al conocimiento para arbustos, acentuándose la diferencia entre escolares y adultos. Es importante destacar aquí que un $86,7 \%$ de los escolares de Porvenir y un 73,3\% de los de Punta Arenas no son capaces de nombrar ni siquiera un árbol. Entre adultos el patrón es opuesto, pues más de $70 \%$ de adultos de las dos localidades nombran más de 2 árboles. De la misma manera que para los otros estratos vegetacionales, los adultos de Punta
Yo sé mucho de plantas vasculares de mi Región
porque tengo contacto con ellas muy a menudo

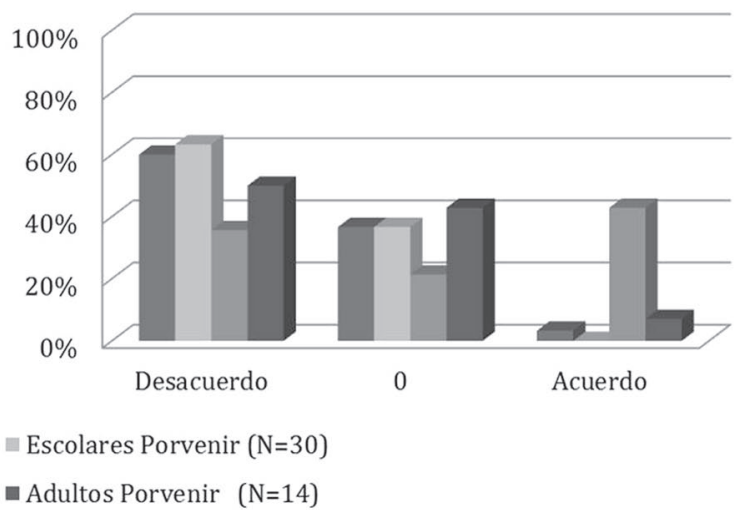

Fig. 1: Escalas de actitud relativas al aprendizaje cotidiano sobre las plantas, evaluadas en grupos de adultos y escolares de Punta Arenas y Porvenir (Tierra del Fuego), Chile. 
TABLA 2: № de especies de plantas citadas por cada grupo de adultos y escolares de Punta Arenas y Porvenir (Tierra del Fuego), Chile, su origen y forma de vida (e.g. árbol, arbusto, hierba). Para Magallanes, las endémicas se distinguieron de las nativas. N= número de citas de plantas.

\begin{tabular}{|c|c|c|c|c|}
\hline & \multicolumn{2}{|c|}{ Escolares } & \multicolumn{2}{|c|}{ Adultos } \\
\hline & $\begin{array}{c}\text { Punta Arenas } \\
(\mathrm{N}=47)\end{array}$ & $\begin{array}{c}\text { Porvenir } \\
(\mathrm{N}=18)\end{array}$ & $\begin{array}{c}\text { Punta Arenas } \\
(\mathrm{N}=124)\end{array}$ & $\begin{array}{c}\text { Porvenir } \\
(\mathrm{N}=95)\end{array}$ \\
\hline $\mathrm{N}^{\circ}$ total de especies & 18 & 9 & 69 & 40 \\
\hline Introducidas & 10 & 3 & 21 & 12 \\
\hline $\begin{array}{c}\text { Nativas o endémicas } \\
\text { de Chile }\end{array}$ & $\begin{array}{c}8 \text { (6 árboles, } 2 \\
\text { arbustos) }\end{array}$ & $\begin{array}{c}6 \text { (3 árboles, } \\
2 \text { arbustos, } 1 \\
\text { hierba) }\end{array}$ & $\begin{array}{c}48 \text { (13 árboles, } 15 \\
\text { arbustos, } 14 \text { hierbas, } \\
5 \text { helechos, } 1 \\
\text { hemiparásita) }\end{array}$ & $\begin{array}{c}28 \text { (8 árboles, } 9 \\
\text { arbustos, } 7 \text { hierbas, } \\
4 \text { helechos) }\end{array}$ \\
\hline $\begin{array}{c}\text { Nativas de } \\
\text { Magallanes citadas }\end{array}$ & $\begin{array}{c}4 \text { (3 árboles, } 1 \\
\text { arbusto) }\end{array}$ & $\begin{array}{l}4 \text { (1 árbol, } 2 \\
\text { arbustos, } 1 \\
\text { hierba) }\end{array}$ & $\begin{array}{c}33 \text { (6 árboles, } 11 \\
\text { arbustos, } 11 \text { hierbas, } \\
5 \text { helechos) }\end{array}$ & $\begin{array}{c}24 \text { (5 árboles, } 8 \\
\text { arbustos, } 7 \text { hierbas, } \\
4 \text { helechos) }\end{array}$ \\
\hline $\begin{array}{l}\text { Endémicas de } \\
\text { Magallanes citadas }\end{array}$ & 0 & 1 (árbol) & $\begin{array}{c}4 \text { (1 árbol, } 1 \text { arbusto, } \\
2 \text { hierbas) }\end{array}$ & $\begin{array}{c}2 \text { (1 árbol, } 1 \\
\text { arbusto) }\end{array}$ \\
\hline
\end{tabular}

Arenas citan más nombres científicos que los de Porvenir. Los árboles citados 2 veces o más por los escolares son lenga (Nothofagus pumilio (Poepp. et Endl.) Krasser), ciprés (Cupressus o Pilgerodendron spp.), araucaria (Araucaria araucana (Molina) K. Koch), pino, coigüe (Nothofagus spp.), manzano (Malus spp.), palmera (Washingtonia robusta $\mathrm{H}$. Wendland) y roble. Los más citados por los adultos son araucaria, canelo (Drimys winteri J.R. et G. Forster), ciprés de las guaitecas (Pilgerodendron uviferum (D. Don) Florin), ciprés, coigüe de Magallanes (Nothofagus betuloides (Mirb.) Oerst.), coigüe, lenga, leña dura (Maytenus magellanica (Lam.) Hook.f.), ñire (Nothofagus antarctica (G.Forst.) Oerst.), notro o ciruelillo (Embothrium coccineum J.R.Forst. \& G.Forst.), pino, roble y sauce (Salix spp.).

Los adultos de Punta Arenas nombran un total de 69 especies de plantas distintas, incluyendo 33 especies nativas de Magallanes, mientras que los adultos de Porvenir nombran 40 especies, incluyendo 24 especies nativas de la región. En cuanto a los escolares, los de Porvenir nombran 9 especies de plantas incluyendo a 3 plantas introducidas, y los de Punta Arenas nombran 18 especies incluyendo a 10 plantas introducidas (Tabla 2).

Conocimiento del uso de las plantas y sus impactos
Como se observa en la Fig. 2, 79\% de los adultos de Porvenir y $64 \%$ de los adultos de Punta Arenas piensan que es beneficioso para la naturaleza que las personas usen las plantas. Los escolares presentan diferencias, con 33\% en Porvenir y 37\% en Punta Arenas en desacuerdo con esta misma afirmación. Por otro lado, la mayoría de las personas (100\% de los adultos de Punta Arenas, 79\% de los adultos de Porvenir, 40\% de los escolares de Punta Arenas y 50\% de los escolares de Porvenir) están en desacuerdo con la afirmación "Yo preferiría que las personas no usaran las plantas".

Entre los escolares, los usos más mencionados son el alimenticio, medicinal y químico, y los menos reconocidos son el simbólico, ornamental, artesanal e industrial. Para los adultos de Porvenir, el uso alimenticio es el más importante, mientras que en Punta Arenas, los usos forrajero y medicinal son más importantes que el alimenticio (Tabla 3). En la categoría "otro", los adultos citan dos tipos de usos: cortavientos y contención de dunas.

Las plantas utilizadas citadas son principalmente introducidas en el caso de los escolares $(71,9 \%$ en Punta Arenas y 54,5\% en Porvenir), mientras que los adultos citan más plantas nativas, muchas de ellas siendo endémicas (Tabla 4). Todas las plantas citadas presentan por lo menos un uso, más de $80 \%$ tienen 2 usos o más, y más de $40 \%$ tienen 5 usos o más. 
Creo que es beneficioso para la naturaleza que las personas usen las plantas

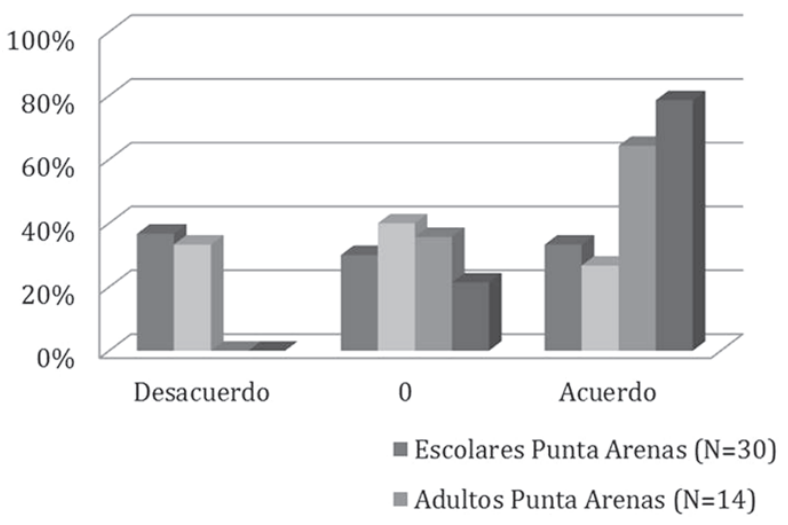

Yo preferiría que las personas no usaran las plantas

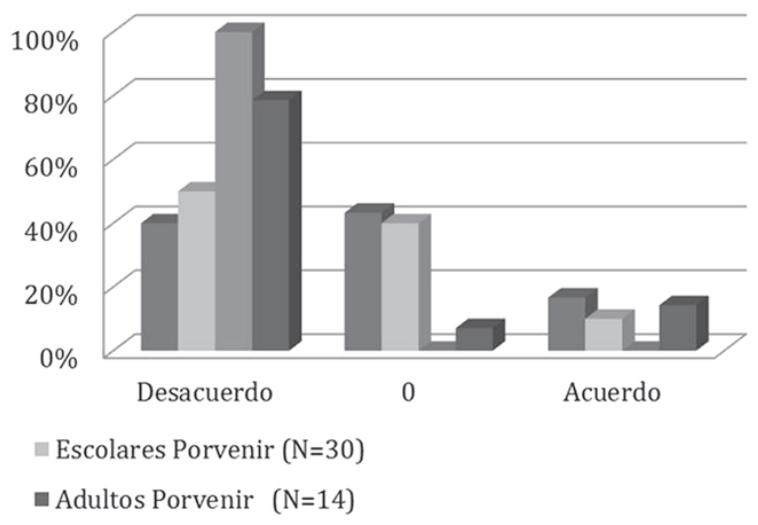

Fig. 2: Escalas de actitud relativas al uso de las plantas por el ser humano y su impacto sobre la naturaleza, según cada grupo de adultos y escolares de Punta Arenas y Porvenir (Tierra del Fuego), Chile.

Más del 50\% de los adultos conoce por lo menos una de las plantas que eran utilizadas por los indígenas de Tierra del Fuego. Sin embargo, el 90\% de los escolares no conocía ninguna de estas plantas.

Valorización de las plantas, de la biodiversidad, y de su conservación

El 21,4\% de los adultos de Punta Arenas participan en una organización ambiental, contra 7,1\% en Porvenir. Por otro lado, el 37\% los escolares de Punta Arenas y el 36\% de los escolares de Provenir afirman realizar actividades en contacto con la naturaleza nunca o una vez al año (20\% una vez al mes en cada ciudad). En el caso de los adultos, $7 \%$ de los individuos de Punta Arenas y 50\% de los individuos de Porvenir realizan actividades en contacto con la naturaleza nunca o solamente una vez al año (43\% una vez al mes en Punta Arenas). Sin embargo, el 100\% de los adultos aprecian estar en contacto con la naturaleza. Esta apreciación es igualmente elevada entre los escolares, tanto en Punta Arenas (90\%) como Porvenir (83\%).

Mientras el 90\% de los escolares están dispuestos a hacer algo para conservar una planta vulnerable, este porcentaje baja (50\% en Punta Arenas y 73,3\% en Porvenir) cuando se les pregunta si pueden hacer alguna contribución específica. Entre los mecanismos o acciones propuestos, se mencionó la conservación directa sobre la planta (60\%), entendida como la no extracción, cuidado, plantación, cultivo en viveros y protección contra los animales. Escolares de Punta Arenas proponen mecanismos de conservación activos, como apoyo a proyectos de conservación (20\%). El 78,6\% de los adultos de Punta Arenas están dispuestos y piensan poder contribuir a conservar una planta vulnerable. En Porvenir, el 86\% de los adultos están dispuestos a hacer algo, pero solamente $57 \%$ de ellos piensan poder contribuir a la conservación de una planta vulnerable.

La valoración de las plantas endémicas es alta, tanto entre adultos (100\%) como escolares (93,3\%), los cuales indican que es importante conservarlas. El 90\% de los escolares de Punta Arenas y el 80\% de los de Porvenir piensa que la conservación de las especies exóticas es importante. En el grupo de adultos, existen diferencia de opinión entre las dos localidades sobre este tema: 29\% en Punta Arenas, contra 93\% en Porvenir, piensan que es importante conservar las introducidas; y 50\% de los adultos de Punta Arenas reconoce el problema de las invasoras, contra 17,8\% en Porvenir.

En el caso de los adultos de Punta Arenas, su decisión es salvar la especie vulnerable (43\%), y la endémica (57\%). Según los que escogen la vulnerable, su conservación es más urgente que la endémica, porque ya está considerada con alto peligro de extinción. En cambio, en Porvenir, 18\% de los adultos escogen la introducida, y 73\% la endémica por ser parte de su patrimonio y ser única. Por otro lado, el 50\% de los escolares de Punta Arenas salvarían a la planta introducida, y el otro $50 \%$ la planta nativa. En Porvenir en cambio, $82 \%$ de los 
escolares salvarían la especie nativa. La motivación para salvar la especie introducida incluye que son más bellas, son de otros países, les llaman más la atención, son únicas o fuera de lo común. Por otro lado, los alumnos que escogen la planta nativa lo hacen porque, según ellos, son de su país, de su región, de su tierra, "tienen un valor medicinal y sentimental", y las exóticas ya están en otros lugares.

La Fig. 3 muestra que más del $80 \%$ de los escolares y más del $90 \%$ de los adultos están en desacuerdo con la afirmación "La existencia de diferentes tipos de plantas no es importante para mi". En cambio, muchos escolares no se pronuncian en cuanto a la afirmación "Si todas las plantas de esta región desparecieran, sus habitantes no podrían sobrevivir", mientras que 79\% de los adultos de Porvenir y 57\% de los adultos de Punta Arenas están de acuerdo con esta afirmación.

El 74\% de los adultos y el 53\% de los escolares de Porvenir, así que el 29\% de los adultos y el 80\% de los escolares de Punta Arenas están de acuerdo con la afirmación "las plantas nativas de nuestro país son igualmente importantes que las exóticas".

El 74\% del total de los individuos, y el 100\% de los adultos de Porvenir, aprueban que la extracción ilegal de plantas debiera ser sancionada.

\section{DISCUSIÓN}

El resultado de las encuestas confirma la hipótesis inicial de existencia de un vacío importante

La existencia de diferentes tipos de plantas no es importante para mi

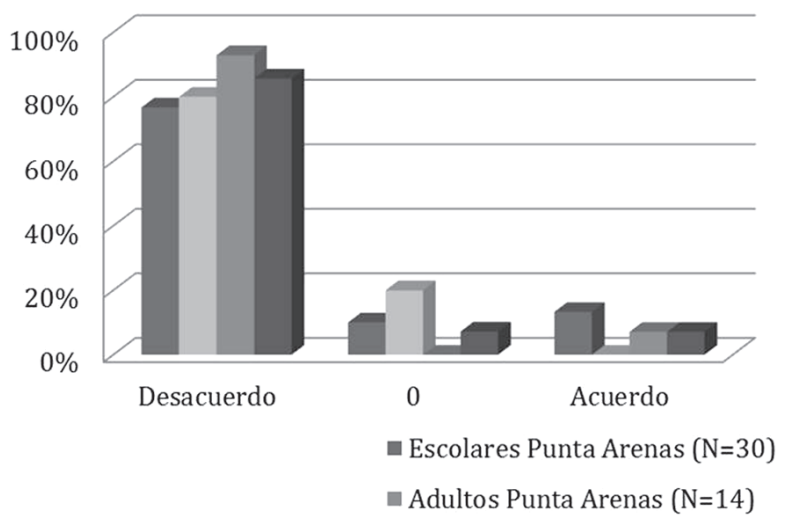

de conocimiento respecto de la vegetación de Tierra del Fuego. Esta brecha resulta especialmente alarmante en el caso de los escolares de ambas localidades y de los adultos de Porvenir.

Conocimiento de la vegetación de Tierra del Fuego

Contrariamente a lo que se podría haber esperado, el conocimiento de plantas no es más alto en Porvenir, lugar más rural que Punta Arenas. En general el conocimiento de las plantas es escaso, hecho que se refleja en todos los grupos entrevistados, a excepción de los Adultos de Punta Arenas, que incluso incluyen información de nombre científico de las plantas. Este resultado refuerza la hipótesis que relaciona un alto conocimiento a su mayor nivel de educación formal, tanto escolar como universitario. Los profesionales que tienen un nivel universitario, la mayoría trabajando en Punta Arenas, presentan un mayor conocimiento de la vegetación. Sin embargo, la encuesta indica que existiría cierto aprendizaje entre el final de la educación media y la vida profesional, lo cual se refleja en el incremento de conocimiento entre vida escolar y adulta. Esto es particularmente interesante, dado el bajo nivel de conocimiento mostrado por escolares, lo que podría indicar precariedad de enseñanza en escuelas locales.

Un alto porcentaje de escolares en Porvenir y Punta Arenas (>60\%), dice tener poco contacto con las plantas, reflejo probablemente de escasas visitas a sitios naturales con el colegio y en el contexto

Si todas las plantas de esta Región desaparecieran, sus habitantes no podrían sobrevivir

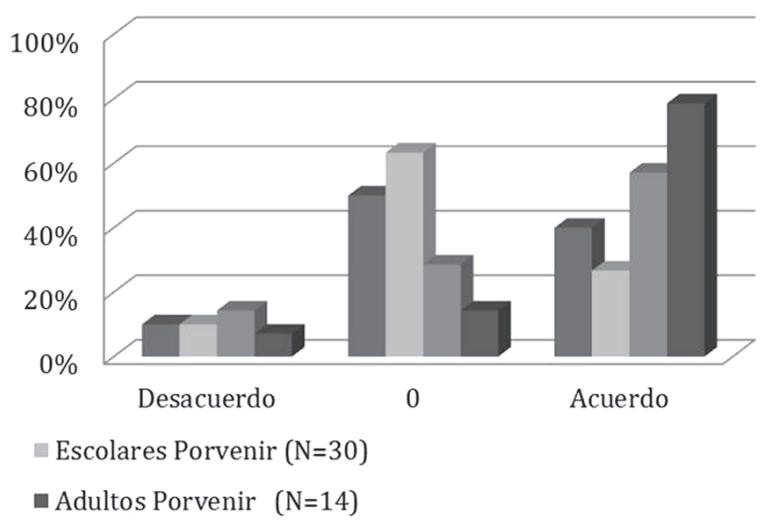

Fig. 3: Escalas de actitud relativas a la valoración de la biodiversidad, y su importancia para la vida humana, obtenidas entre grupos de adultos y escolares de Punta Arenas y Porvenir (Tierra del Fuego), Chile. 
TABLA 3: Tipo de uso por especie entre las diferentes plantas utilizadas por la población local de Magallanes, citadas dos veces o más en cada grupo de adultos y escolares de Punta Arenas y Porvenir (Tierra del Fuego), Chile.

\begin{tabular}{|c|c|c|c|c|}
\hline Plantas & Nombre científico & Usos & Escolares & Adultos \\
\hline Apio austral & Apium australe Thouars & Alimenticio, Medicinal & & $\mathrm{X}$ \\
\hline Calafate & $\begin{array}{c}\text { Berberis microphylla } \mathrm{G} . \\
\text { Forst. }\end{array}$ & $\begin{array}{l}\text { Alimenticio, Medicinal, } \\
\text { Artesanal, Leña }\end{array}$ & $\mathrm{X}$ & $\mathrm{X}$ \\
\hline Canelo & $\begin{array}{c}\text { Drymis winteri J.R. et } \\
\text { G.Forst. }\end{array}$ & Medicinal & & $\mathrm{X}$ \\
\hline Coirón & Festuca magellanica Lam. & Forrajero & & \\
\hline Hierba buena o menta & Mentha x piperita $\mathrm{L}$. & Medicinal, Alimenticio & $\mathrm{x}$ & \\
\hline Lenga & $\begin{array}{l}\text { Nothofagus pumilio } \\
\text { (Poepp. et Endl.) Krasser }\end{array}$ & $\begin{array}{c}\text { Madera, Construcción, } \\
\text { Artesanal, Medicinal, } \\
\text { Leña }\end{array}$ & & $\mathrm{X}$ \\
\hline Manzanilla & $\begin{array}{c}\text { Chamomilla } \\
\text { suaveolens(Pursh) Rydb. }\end{array}$ & Medicinal, Alimenticio & $\mathrm{X}$ & \\
\hline Matico & Buddleja globosa Hope & Medicinal & $\mathrm{X}$ & \\
\hline Orégano & Origanum vulgare $\mathrm{L}$. & Medicinal, Alimenticio & $\mathrm{X}$ & \\
\hline Perejil & $\begin{array}{l}\text { Petroselinum crispum } \\
\text { (Mill.) Fuss }\end{array}$ & Alimenticio & $\mathrm{X}$ & \\
\hline Repollo & Brassica oleracea L. & Alimenticio & & $\mathrm{X}$ \\
\hline Rosa & Rosa spp. & Ornamental & $\mathrm{X}$ & \\
\hline Ruibarbo & Rheum rhabarbarum $\mathrm{L}$. & Alimenticio & $\mathrm{x}$ & $\mathrm{x}$ \\
\hline Total & & & 8 & 6 \\
\hline
\end{tabular}

familiar.

Esto sorprende pues la Región de Magallanes es por lejos la que presenta mayor superficie protegida en Chile (Ministerio del Medio Ambiente, 2011), por lo que se deduce que hay escaso acceso a estas zonas por parte de la población local. Por lo tanto, la diferencia de conocimiento entre escolares y adultos se podría explicar también por el hecho de que los adultos, cuando niños, salían más al campo que los niños de las nuevas generaciones.

Cuando se les pide nombres de hierbas a los encuestados, más de $80 \%$ de los escolares no son capaces de nombrar ni siquiera una hierba, lo que refleja un gran vacío de conocimiento a este nivel. En cuanto a los errores cometidos por los escolares cuando citan árboles o arbustos en vez de hierbas, para algunas de estas especies (e.g. boldo, matico, rosa mosqueta), es probable que el hecho de que sean hierbas para infusión o "té de hierbas" les haya inducido a error.

Acerca del conocimiento sobre los árboles, los errores que incurren los escolares consisten en calificar árboles como "fauna", afirmar que crecen araucarias en Viña del Mar (Región de Valparaíso) y en Magallanes (Región de Magallanes y Antártica chilena), y que el pino (Pinus spp.) y el roble (Nothofagus obliqua (Mirb.) Oerst.) son nativos de Magallanes. Algunos adultos también indican que el roble crece en Magallanes.

En una Región donde existen sólo 7 especies de árboles contra más de 20 tipos de helechos, la mayor parte de los escolares no sabe decir si hay más especies de árboles o helechos en Magallanes, y muchos preguntaron qué era un helecho durante la encuesta, lo que refleja la falta de conocimiento en aspectos básicos de botánica entre este grupo, especialmente en relación al conocimiento de biodiversidad de plantas de menor tamaño.

El conocimiento de la flora nativa se reduce en Punta Arenas, en comparación a Tierra del Fuego, fenómeno observado especialmente entre el grupo de escolares. A pesar que en Porvenir los escolares nombran pocas plantas $(\mathrm{N}=18)$, un amplio porcentaje de ellas (78\%) son nativas o endémicas 
TABLA 4: Proporciones de citas de plantas utilizadas de diversos orígenes: introducidas, nativas o endémicas de Chile, nativas o endémicas de Magallanes entre grupos de adultos y escolares de Punta Arenas y Porvenir (Tierra del Fuego), Chile. Entre paréntesis № de especies citadas.

\begin{tabular}{ccccc}
\hline & \multicolumn{2}{c}{ Escolares } & \multicolumn{2}{c}{ Adultos } \\
\hline Origen de las plantas & Punta Arenas & Porvenir & Punta Arenas & Porvenir \\
Introducidas & $71.9(13)$ & $54.5(7)$ & $20.7(6)$ & $32.0(4)$ \\
Nativas/endémicas de Chile & $28.1(3)$ & $45.5(4)$ & $79.3(7)$ & $68.0(9)$ \\
Nativas/endémicas de Magallanes & $21.9(1)$ & $36.4(3)$ & $75.9(6)$ & $68.0(9)$ \\
\hline
\end{tabular}

de la Región de Magallanes y Antártica chilena. Los adultos no muestran diferencias importantes, y en ambos sitios, existe un importante porcentaje de especies exóticas nombradas (30\%) (Tabla 2).

Conocimiento del uso de las plantas y sus impactos

Contrario a lo esperado, un alto porcentaje de los encuestados piensa que el uso de las plantas tiene un impacto positivo en la naturaleza. Esta respuesta fue justificada por la mayoría indicando la provisión de servicios ecosistémicos. En Punta Arenas sin embargo, más del 60\% de los adultos consideran que el uso podría ser positivo a condición de ser sustentable (e.g. buen plan de manejo, educación). Los escolares mayoritariamente indican que el efecto del uso podría ser negativo o sin impacto sobre la naturaleza y justifican su respuesta considerando el agotamiento de los recursos y su mal manejo.

La apreciación de los usos de las plantas de Tierra del Fuego cambia dependiendo del grupo y del lugar en que éste se encuentre. Se destaca que contrario a lo esperado, en Punta Arenas se valoriza más el uso simbólico que en Porvenir. Ello a pesar de que la población naturalmente heredera de la tradición indígena de Tierra del Fuego es la de la Isla, la cual se concentra en Porvenir. El conocimiento sobre el uso tradicional o indígena de la flora de Tierra del Fuego es escaso. Este resultado refleja una pérdida importante de conocimiento del patrimonio cultural de la Isla, y una falta de respuesta frente a esta pérdida por parte de los sistemas educativos actuales. Es esperable que este patrón mejore, luego de un trabajo educativo activo de rescate del patrimonio cultural.
La falta de conocimiento del uso tradicional de la vegetación se confirma al analizar la identidad de las especies mencionadas durante la encuesta (Tabla 5). Por ejemplo, se menciona bambú (Bambu savulgaris Schrad. ex Wendl.o Chusquea quila Kunth) y roble (Nothofagus obliqua), especies que no existen en Tierra del Fuego, por lo que el conocimiento de su uso, no deriva de la zona. Es probable que sea información aprehendida del uso dado por mapuches, los cuales habitan varias centenas de kilómetros más al norte de Magallanes. Esta hipótesis se sustenta en la mención al canelo, árbol sagrado para los mapuche. Este patrón puede encontrar su explicación última, en el hecho que mucha de la población actual de Magallanes deriva de inmigración chilota (Martinic, 1999), isla donde predomina esta cultura. En cualquier caso, el rescate de este conocimiento patrimonial representa una tarea interesante para los diseños educativos en esta parte del país.

A pesar de que los individuos reconocen diversos usos a la vegetación, el uso alimenticio, constituye el más ampliamente reconocido en Magallanes, siendo mencionados otros como el medicinal, forrajero y maderero. Los profesionales de Punta Arenas reconocen en las plantas usos amplios, que incluyen incluso educación, mantención de ecosistemas sanos y con alta resiliencia, protección del suelo y contribución al equilibrio de la naturaleza. En Porvenir por el contrario, sólo los escolares mencionan oxigenación y producción de tierra de hojas.

El hecho de que todas las plantas citadas presenten por lo menos un uso indica que las personas aprenden a conocer las plantas cuando presentan 
TABLA 5: Plantas que utilizaban los indígenas de Tierra del Fuego, según cada grupo de adultos y escolares presentes en Punta Arenas y Porvenir (Tierra del Fuego), Chile. Los usos mencionados por los cuatro grupos están anotados en la columna usos.

\begin{tabular}{|c|c|c|c|c|}
\hline Plantas & Nombre científico & Usos & Escolares & Adultos \\
\hline Apio austral & Apium australe Thouars & Alimenticio & & \\
\hline Bambú & $\begin{array}{l}\text { Bambus avulgaris Schrad. Ex } \\
\text { Wendl. o Chusquea quila Kunth }\end{array}$ & Artesanal & $\mathrm{X}$ & \\
\hline Calafate & Berberis microphylla G. Forst. & $\begin{array}{l}\text { Alimenticio, Medicinal, } \\
\text { Artesanal, Símbolo }\end{array}$ & $\mathrm{X}$ & $\mathrm{X}$ \\
\hline Canelo & Drimys winteri J.R. et G.Forster & Medicinal, Símbolo & $\mathrm{X}$ & $\mathrm{X}$ \\
\hline Ciprés & $\begin{array}{l}\text { Pilgerodendron uviferum (D. } \\
\text { Don.) Florin }\end{array}$ & Construcción & $\mathrm{X}$ & \\
\hline Coigue & $\begin{array}{c}\text { Nothofagus betuloides (Mirb.) } \\
\text { Oerst. }\end{array}$ & $?$ & & $\mathrm{X}$ \\
\hline Coirón & Festuca magellanica Lam. & Construcción & & $\mathrm{X}$ \\
\hline $\begin{array}{l}\text { Frutilla } \\
\text { magallánica }\end{array}$ & Rubus geoides J.E.Sm. & Alimenticio & & $\mathrm{X}$ \\
\hline Junquillo & $\begin{array}{l}\text { Marsippospermum grandiflorum } \\
\text { (L.f.) Hook. }\end{array}$ & Artesanal & & $\mathrm{X}$ \\
\hline Lenga & $\begin{array}{c}\text { Nothofagus pumilio (Poepp. et } \\
\text { Endl.) Krasser }\end{array}$ & Cortavientos, Leña & & $\mathrm{X}$ \\
\hline Murtilla & Empetrum rubrumVahl ex Willd. & Alimenticio & & $\mathrm{X}$ \\
\hline Musgos y ramas & - & Construcción & $\mathrm{X}$ & \\
\hline Nalca & Gunnera tinctoria (Molina) Mirb. & Alimenticio & & $\mathrm{x}$ \\
\hline $\begin{array}{l}\text { Pasto parecido a } \\
\text { cola de zorro }\end{array}$ & Hordeum spp. & Artesanal & & \\
\hline Roble & Nothofagus obliqua (Mirb.) Oerst. & Construcción & $\mathrm{X}$ & \\
\hline Romaza & Rume xcrispus L. & Medicinal & & $\mathrm{X}$ \\
\hline Siete venas & Plantago lanceolata L. & ? & & $\mathrm{x}$ \\
\hline Zarzaparrilla & $\begin{array}{l}\text { Ribes magellanicum } \\
\text { Poir. }\end{array}$ & $?$ & & $X$ \\
\hline Total & & & 6 & 12 \\
\hline
\end{tabular}

alguna utilidad específica para la sociedad humana. Cuando se analiza el conocimiento del uso por especie, se constata que sólo el calafate (especie nativa) y el ruibarbo (especie exótica), ambas ampliamente utilizadas en la elaboración de repostería, son las que tienen mayor grado de reconocimiento entre los diferentes grupos analizados (Tabla 3).

En general, el conocimiento del uso de las plantas se refiere a aquellas plantas alimenticias $y$ medicinales que se encuentran en el comercio, o plantas cultivadas. Esto se refleja claramente en la elevada proporción de citas de especies introducidas entre escolares de Punta Arenas (Tabla 4). Los escolares de Porvenir es el grupo que menor conocimiento tiene sobre los usos de la flora local. En cambio, los adultos citan más plantas nativas que introducidas, y casi todas nativas o endémicas de Magallanes. Este resultado revela el gran potencial para el aprendizaje de la flora que es posible de ser alcanzado entre la población escolar de Punta Arenas y Tierra del Fuego.

Valorización de las plantas, de la biodiversidad y de su conservación

Las proporciones de adultos que participan en una organización ambiental son bajas, aunque más altas en Punta Arenas donde muchos de los 
profesionales no son nativos de la región. Por otro lado, el contacto de los grupos encuestados con la naturaleza es escaso, existiendo un porcentaje importante de personas que reconoce que nunca o sólo una vez al año experimentan esa experiencia. Esta falta de contacto efectivo contrarresta con el hecho que el $100 \%$ de los adultos y más del $80 \%$ de los escolares aprecian estar en contacto con la naturaleza. El grupo de adultos profesionales de Punta Arenas es el que reconoce mayor contacto con la naturaleza. Este grupo está compuesto por profesionales que viven en la región recientemente. Entre los escolares destaca el hecho que tienen poco contacto con la naturaleza. Esto es un interesante factor a considerar al momento de desarrollar políticas o herramientas educacionales en Magallanes, pues cuando se espera promover educación ambiental, parece ser importante aprovechar las oportunidades que se asocian a visitas de campo o trabajos en terreno, sobre todo considerando la significativa superficie bajo protección que existe en la región.

El valor de la acción para la recuperación de las plantas con problemas de conservación se reconoce entre los grupos encuestados, sin embargo no tienen claro qué acción puede ser desarrollada con ese fin. Entre los adultos, su capacidad de respuesta fue percibida como mayor, dado que un alto porcentaje de los adultos están dispuestos y piensan poder contribuir a conservar una planta vulnerable, pero sin siempre tener claro cómo podrían actuar. Es interesante destacar que un 50\% de los adultos propone como mecanismo de conservación informar, informarse, educar y valorar mejor los recursos.

La valoración de las plantas endémicas es alta. Se reconoce en este objetivo la unicidad de estas especies y el valor patrimonial de ellas, especialmente entre los grupos entrevistados de Porvenir comparados con los de Punta Arenas (23\% $\mathrm{v} / \mathrm{s} 27 \%$ para escolares, $21,5 \% \mathrm{v} / \mathrm{s} 36 \%$ para adultos respectivamente).

Los escolares de Punta Arenas y de Porvenir piensan que la conservación de las especies exóticas es importante. Según ellos, estas plantas "tienen derecho a vivir como las otras" y en general "son muy bonitas". En el grupo de adultos, existe diferencia de opinión entre las dos localidades sobre este tema, con una mayor valoración de las plantas introducidas en Porvenir. Es probable que nuevamente el nivel de estudio de los adultos de Punta Arenas sea el factor que explique este patrón.
Para la mayor parte de la población, la existencia de diferentes tipos de plantas es importante (Fig. 3). Por otro lado, más del 50\% de los adultos aprueban que si todas las plantas de su región desaparecieran, sus habitantes no podrían sobrevivir. En cambio, los escolares no saben decidirse frente a esta afirmación, y la mayoría no está ni de acuerdo, ni en desacuerdo. Es probable que en este último caso, la respuesta se deba a que muchos escolares no conocen la relación de dependencia que existe entre la vida humana y las plantas, hecho que puede ser revertido con estrategias educacionales ad-hoc.

Con excepción de los adultos de Punta Arenas, gran proporción de la población está de acuerdo con la afirmación "las plantas nativas de nuestro país son igualmente importantes que las exóticas", y en desacuerdo con la idea de tener que eliminar las plantas exóticas por la simple razón que causan daño a las nativas. Esta apreciación refleja la falta de conocimiento sobre los efectos ecológicos, económicos y sociales que ejercen las especies exóticas sobre los ecosistemas nativos, siendo consideradas a nivel mundial como la segunda causa más importante de pérdida de biodiversidad (IUCN, 2011). Refleja asimismo, el desconocimiento del problema que significa la presencia de especies exóticas en Tierra del Fuego, las cuales tienen efectos sobre la calidad del forraje, la mantención de procesos ecosistémicos, y sobre diferentes atributos de la biodiversidad local.

Existe un interés más allá de la acción de conservación de la flora, de sancionar a las personas que realizan actividades que dañan a la vegetación. La mayoría de los encuestados aprueban que la extracción ilegal de plantas debiera ser sancionada. Esto es importante al momento de considerar medidas aleccionadoras, pues los pobladores locales reconocen que es importante controlar la extracción de las plantas y de castigarla cuando ella no es sustentable.

\section{CONCLUSIONES}

Logramos recuperar información suficiente para caracterizar la población e incluso observar tendencias y patrones. Este estudio no pretende ser estadísticamente representativo, sino que muestra ciertas tendencias entre variables, las cuales en un estudio futuro podrían corroborarse utilizando una muestra mayor.

Según Nisbet et al. (2009), "la educación ambiental y las experiencias en contacto con la 
naturaleza permiten aumentar la conexión entre el ser humano y la naturaleza". Investigaciones sobre programas de aventura al aire libre y terapias basadas en la naturaleza demostraron que un cambio de perspectiva, de opinión y de sensibilidad a nivel de las relaciones entre el ser humano y la naturaleza es posible (Nisbet et al., 2009). WCS podría ser una de las instituciones encargadas de provocar este cambio en la región de Magallanes, con el fin de crear y mantener un equilibrio entre el desarrollo humano, el de la naturaleza y el uso de sus recursos.

El conocimiento de la vegetación de Tierra del Fuego es escaso en Magallanes. Los niños nombran pocas plantas, y el conocimiento de los adultos, a pesar de que incluyen más especies, sigue siendo escaso en comparación al número total de especies de plantas existentes en la región. El futuro desafío consistirá en formar localmente a escolares y profesionales de la región, los que puedan conocer de primera mano la vegetación que les es propia.

En los colegios de ambas localidades, el nivel de educación sobre la flora en general y sus funciones en la naturaleza resulta ser muy bajo. Un programa de educación debería comenzar con temas muy básicos, tales como las diferencias entre hierbas, helechos, arbustos y árboles, sus hábitats respectivos, su diversidad en la Región de Magallanes y comparativamente a las otras regiones de Chile, por nombrar algunas. Los niños podrían participar activamente de los programas de conservación, aprendiendo a valorizar la existencia de la naturaleza de su Región, el contacto y la relación que pueden tener con ella, y dándose cuenta que estos recursos naturales tienen muchos valores culturales, éticos, ecológicos y económicos.

Este estudio ha sido limitado a las plantas vasculares, dejando de lado otros recursos vegetales y animales. Sería interesante indagar con una metodología similar respecto del conocimiento y valoración de la fauna de la Región de Magallanes. Es posible aventurar que ésta tendría probablemente el mismo tipo de resultados, con un vacío importante de conocimiento sobre la fauna nativa, y una mayor valorización de las especies exóticas. Por otro lado, sería interesante realizar otros estudios que puedan comparar el nivel de conocimiento y valoración entre escolares de colegios públicos y escolares de colegios particulares, así que el nivel de conocimiento y valoración entre poblaciones rurales y urbanas.
El análisis del conocimiento y uso de la vegetación de Karukinka es pionero en la región. Este trabajo entrega herramientas prácticas con base científica para avanzar en la conservación de Karukinka en Tierra del Fuego.

Este trabajo permite poner a prueba la hipótesis inicial de la existencia de una gran brecha de conocimiento y valoración de la flora de Tierra del Fuego dentro de la población local, y especialmente en el caso de los escolares y adultos de Porvenir. A futuro, la realización de proyectos de educación ambiental se podría realizar con prioridad en estos dos grupos.

\section{AGRADECIMIENTOS}

Al equipo WCS Chile, a los directores de los colegios, a los profesionales de Punta Arenas y Porvenir, a los profesores Miguel Gómez y Paulina Fernández de la Pontificia Universidad Católica de Chile, a Gonzalo Ossa Gómez.

\section{LITERATURA CITADA}

Albuquerque, U.P., Sousa Araújo, T.A., Alves, R.M., Teixeira Do Nascimento, V., Farias, P.L.R., Marcelino, M.J., Leal, A.N., \& Lima, A.E. (2009). How ethnobothany can aid biodiversity conservation: reflections on investigations in the semi-arid región of NE Brazil. Biodiversity Conservation, 18, 127-150.

Azócar de la Cruz (2009). Análisis de Datos Clase I. Uso básico de spss, procesamiento y análisis descriptivo de datos.

Bateman, I., Carson, R., Day, B., Hanemann, M., Hanley, N., Hett, T., Jones-Lee, M., Loomes, G., Mourato, S., Özdemiroglu, E., Pearce Obe, D.W., Sugden, R., \& Swanson, R. (2002). Economic Valuation with Stated Preference Techniques: A Manual. United Kingdom, Cheltenham: Edward Elgar Publishing.

Bermúdez, A., Oliveira-Miranda, M.A., \& Velázquez, D. (2005). La investigación etnobotánica sobre plantas medicinales: una revisión de sus objetivos y enfoques actuales. Interciencia, 30(8), 453-459.

Castetter, E.F. (1944). The Domain of Ethnobiology. The American Naturalist, 78 (775), 158-170. Chapman, A. (1986). Los selk'nam: la vida de los 
onas. Buenos Aires: Ed. Emecé.

Ministerio del Medio Ambiente (2011). Las áreas protegidas de Chile. http://www.mma.gob. cl/1257/articles-50613_pdf.pdf

De Gregorio, J. (2009). El crecimiento en Chile y el cobre. Preparado para la conferencia de Conmemoración CESCO 25 años "19842009, 25 Años de la Industria del Cobre y su impacto en Chile", 1ำ de septiembre, Santiago, Chile. Véase la página web: http://www.cesco. $\mathrm{cl} /$ assets/docs/estudios-y-presentaciones/ Jose_De_Gregorio.pdf

Elejabarrieta, F.J., \& Iñiguez, L. (1984). Construcción de escalas de actitud, tipo Thurstone y Likert. Universidad Autónoma de Barcelona. Véase la página web: http://revinut.udea.edu.co/ index.php/ceo/article/viewFile/6820/6246

Fischer, A., \& Van Der Wal, R. (2007). Invasive plant suppresses charismatic seabird - the construction of attitudes towards biodiversity management options. Biological Conservation, 135, 256-267.

Fischer, A., \& Young, J.C. (2007). Understanding mental constructs of biodiversity: Implications for biodiversity management and conservation. Biological Conservation, 136, 271-282.

INE (2012). Resultados XVIII Censo de Población 2012. http://www.emol.com/documentos/ archivos/2013/04/02/20130402145438.pdf

IUCN (2011). Especies invasoras. http://www. iucn.org/es/sobre/union/secretaria/oficinas/ sudamerica/sur_trabajo/sur_especies/sur_ invasoras/

Ladio, A. (2007). Curso "Elaboración de proyectos etnobotánicos”. INIBIOMA-Universidad Nacional del Comahue.

Martinic, M. (1999). La inmigración chilota en Magallanes. Apreciación histórica sobre sus causas, características y consecuencias, en Anales del Instituto de la Patagonia, Serie Ciencias Sociales, 27, 27-47.

Meyerhoff, J. \& Liebe, U. (2006). Protest beliefs in contingent valuation: Explaining their motivation. Ecological Economics, 57, 583594.

Ministerio de agricultura, 2008. Reglamento general de ley sobre recuperación del bosque nativo $y$ fomento forestal.

Nisbet, E.K., Zelenski, J.M., \& Murphy, S.A. (2009). The Nature Relatedness Scale: Linking Individuals' Connection with Nature to Environmental Concern and Behavior. Environment and Behavior, 41(5), 715-740.

Ocampo, S.R.A. (1994). Etnobotánica y etnofarmacología, disciplinas de valor en la domesticación de plantas. Proyecto de Conservación para el Desarrollo Sostenible en América Central, CATIE. http://www.fao. org/docrep/T2354S/t2354s0h.htm, visitado en octubre del año 2011.

Otero, L. (2006). La huella del fuego: Historia de los Bosques Nativos, Poblamiento y cambios en el paisaje del sur de Chile. Santiago de Chile: Ed. Pehuén.

Pacheco, B.J., Casado, Y.S., \& Nunez, L.L. (2007). Algoritmos meméticos para selección de variables en el análisis discriminante. Estadística española, 49(165), 333-347.

Quivy, R., \& Van Campenhoudt, L. (2006). Manuel de recherche en sciences sociales. Paris: Dunod.

Secretaría del Convenio sobre la Diversidad Biológica (2009). Informe sobre la Conservación de las Especies Vegetales: Una revisión de los progresos realizados en la aplicación de la Estrategia Mundial para la Conservación de Plantas (GSPC).

Seltiz, C., Jahoda, M., Deutsh, M., \& Cook, S. (1965). Métodos de investigación en las relaciones sociales. Madrid: Ed. Ruallp.

Sernatur (2011). Tierra del Fuego. http://www. sernatur.cl/internacional/destino.php? $\mathrm{n}=16$

SUBDERE (2009a). Punta Arenas. http://www. subdere.gov.cl/1510/propertyvalue-25024. html

SUBDERE (2009b). Porvenir. http://www.subdere. gov.cl/1510/propertyvalue- 25028.html

Universidad de Chile (2000). Informe país, estado del medio ambiente en Chile - 1999. Area de Desarrollo Sustentable, Universidad de Chile. Departamento de Políticas Públicas.

WCS Chile (2009). http://www.karukinkanatural.cl 
